\title{
Weniger macht Angst
}

\section{Anna Sax}

Lic oec. publ., MHA, Mitglied der Redaktion

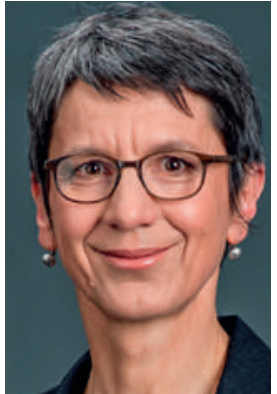

Wer in der Gesundheitsversorgung nach Ineffizienzen und Fehlanreizen fahndet, wird mit grösster Wahrscheinlichkeit fündig: In der Medizin geschieht Unnötiges am Laufmeter. Es wird zu viel diagnostiziert, zu viel therapiert und zu viel operiert. Ein knappes Jahrzehnt nach den engagierten Debatten über Rationierung im Gesundheitswesen lautet nun der neue Zauberspruch: «Weniger ist mehr». Das SAMW-Symposium 2015 zur Versorgungsforschung* widmete sich unter anderem der Frage, was Versorgungsforschung zur Lösung des Problems der Überversorgung beitragen könne.

Nikola Biller-Andorno, Professorin am Institut für biomedizinische Ethik der Universität Zürich, machte den Anfang mit einem Statement über Appropriateness. Sie fragte sich zunächst, was unter Appropriate care, zweckmässiger Versorgung, zu verstehen sei, und postulierte als Arbeitsdefinition das Ziel-Dreieck Bevölkerungsgesundheit, Pro-Kopf-Ausgaben und Experience of care. Diese drei Ziele gelte es im Auge zu behalten, damit Gesundheitsversorgung zweckmässig erfolgen könne, betonte Biller-Andorno. Die ersten beiden Ziele sind unschwer zu verstehen. Die Suche nach einer

Wer definiert eigentlich, was appropriate, also zweckmässig und angemessen ist?

Übersetzung für den Begriff Experience of care zeigt jedoch, wie schwierig dieses Thema tatsächlich zu vermitteln ist. Der britische nationale Gesundheitsdienst NHS stellt auf seiner Website ziemlich lapidar fest: "Experience of care means many things to many people." Aus den weiteren Ausführungen des NHS schliesse ich, dass es irgendwie darum geht, wie die Menschen mit dem Gesundheitssystem zurechtkommen, welche Erfahrungen sie darin machen und wie es ihnen dabei hilft, gesund zu werden oder zu bleiben. Und so kamen die Referentin und ihr Publikum ins Grübeln: Wer definiert eigentlich, was appropriate, also zweckmässig und angemessen ist? Wer sind die Stakeholders, die es zu bedienen gilt? Natürlich, es geht um die Patienten, wie konnten wir das nur vergessen! Aber sind es nur diese oder auch die (noch) Gesunden, also die potentiellen Patientinnen, also die ganze Bevölkerung? Und woher erhält die Bevölkerung ihre Informationen? Bekanntlich kann die öffentliche Meinung manipuliert werden, Biller-Andorno führte als Beispiele ScreeningProgramme oder die Komplementärmedizin auf. Sie verlangte nach Evidenz und nach Studien, und zwar unbiased. Ich fragte mich indessen: Was ist, wenn die Leute gar keine zweckmässige Gesundheitsversorgung haben wollen?

\section{Warum also, fragte ich mich zum wiederholten} Mal, geschieht eigentlich nicht mehr?

Erste Hinweise auf Antworten lieferte Steve Pearson, Bioethik-Professor aus Bethesda (USA). Auch er ortete waste, Verschwendung, wohin das Auge reicht. Pearson erklärte, weshalb es so schwierig sei, gegen besseres Wissen auf Überflüssiges zu verzichten: Evidenzbasierte Reduktion führe zu one-size fits all, d.h. zu einer Einheitsgrösse, und verkenne das Bedürfnis der $\mathrm{Pa}$ tientinnen, als Einzelfall wahrgenommen zu werden. Professor Nicolas Rodondi vom Inselspital warb für das Projekt Smarter Medicine der SGIM. Dieses greift Interventionen heraus, die häufig vorgenommen werden, jedoch keinen nachweisbaren Nutzen bringen und deshalb in Zukunft zu vermeiden sind. Viele davon sind Leistungen, an die sich die Patientinnen gewöhnt haben und die ihr Vertrauen zum Arzt festigen. Versorgungsforschung sei, liess der krankheitshalber abwesende Professor Gianfranco Domenighetti ausrichten, in der Schweiz «äusserst unpopulär».

Am Symposium waren viele versammelt, die im Gesundheitssystem etwas zu sagen haben, sprich, die etwas verändern könnten, wenn sie wirklich wollten: Chefärzte, Professorinnen, Forscher, Vertreterinnen von kantonalen und nationalen Behörden, Versicherungen, Spitälern und Industrie. Warum also, fragte ich mich zum wiederholten Mal, geschieht eigentlich nicht mehr? Und endlich, ganz am Ende in der Podiumsdiskussion, als etwa ein Viertel der Teilnehmenden bereits aufgebrochen war, kam mit Bea Heim doch noch eine Patientenvertreterin zu Wort. Was sie sagte, war so etwas wie die ultimative Antwort auf meine Fragen. Heim sagte: «Less is more - genau davor fürchten sich die Patientinnen und Patienten.» 